\title{
Preliminary empirical models for predicting shrinkage, part geometry and metallurgical aspects of Ti-6Al-4V Shaped Metal Deposition builds
}

\author{
Gustavo Escobar-Palafox *, Rosemary Gault and Keith Ridgway \\ AMRC, University of Sheffield, Advanced Manufacturing Park, Wallis Way, \\ Catcliffe, Rotherham, S60 5TZ, UK \\ *corresponding author: g.escobar@amrc.co.uk
}

\begin{abstract}
Shaped Metal Deposition (SMD) is an additive manufacturing process which creates parts layer by layer by weld depositions. In this work, empirical models that predict part geometry (wall thickness and outer diameter) and some metallurgical aspects (i.e. surface texture, portion of finer Widmanstätten microstructure) for the SMD process were developed. The models are based on an orthogonal fractional factorial design of experiments with four factors at two levels. The factors considered were energy level (a relationship between heat source power and the rate of raw material input.), step size, programmed diameter and travel speed. The models were validated using previous builds; the prediction error for part geometry was under $11 \%$. Several relationships between the factors and responses were identified. Current had a significant effect on wall thickness; thickness increases with increasing current. Programmed diameter had a significant effect on percentage of shrinkage; this decreased with increasing component size. Surface finish decreased with decreasing step size and current.
\end{abstract}

\section{Introduction}

Titanium alloys are commonly used in the aerospace industry due to their combined properties, such as high strength to weight ratio, corrosion resistance, excellent mechanical properties and capacity to withstand high temperatures [1]. However, their use is strongly limited by its higher cost relative to competing materials, such as aluminium alloys and steels. One of the most widely used titanium alloys is the two-phase $(\alpha+\beta)$ Ti-6Al-4V alloy, accounting for $60 \%$ of the total titanium production [2].

Additive manufacturing has attracted much interest in recent years for the capability to reduce manufacturing times and materials costs [3]. In additive manufacturing parts are built layer by layer directly from a CAD model. The process involves a heat source, which could be a laser or electron beam or a welding arc, and the inclusion of raw material, in either powder [4, 5] or wire form [6-8]. An automatic manipulator drives the power unit according to a computer generated path calculated from a CAD model. Different geometries can be built by varying the manipulator path, the heat source power and the rate of raw material fed into the power source.

An important factor affecting part geometry is shrinkage. Shrinkage induces a negative volume variation during cooling from the molten to the solid state at room temperature; and has to be compensated for, if a desired geometry is to be achieved. The percent of shrinkage is determined by the initial temperature of the molten metal, its chemical composition and possibly by the conditions of its solidification (cooling rate and component shape). 
Furthermore, the microstructure and properties of titanium alloys additive manufactured parts are closely linked to the layer generation process and parameters [9]. Additive manufacturing processes induce a repetitive cooling and heating in the material affecting the final microstructure.

This article provides details of experiments carried out with the aim of building an empirical model able to predict shrinkage, part geometry, surface finish, and portion of finer Widmanstätten microstructure. The model was developed for shaped metal deposition (SMD), an additive manufacturing process, which uses a TIG welding unit as a power source, wire as raw material and a six-axis robot as a manipulator. The models were built for the titanium alloy Ti-6Al-4V.

\section{Methodology}

\subsection{Shaped Metal deposition}

The experiments were carried out on an SMD cell. The cell consists of a 6-axis robot linked to a 2axis rotary table. The system uses cold wire TIG deposition with a Tungsten cathode immersed in an inert argon atmosphere (Ar, 99.999\% purity). This prevents the substrate, electrode and part from reacting with atmospheric gasses. Ti-6Al-4V grade 5 wire with a diameter of $1,2 \mathrm{~mm}$ is injected into the chambers through an annular argon feed pipe. The depositions are monitored via a water cooled vision system so the welding arc (electrode, contact tip) can be controlled by altering the wire feed.

The experiments in this study were performed using Ti-6Al-4V substrate plates that were $250 \mathrm{~mm}$ wide by $250 \mathrm{~mm}$ long by $20 \mathrm{~mm}$ thick. The substrate plate was fixture in the middle of the rotary table. An additional plate was set-up on the side and used as an oxygen burn-of plate (Figure 1). The parts consisted in cylinders of different diameters $(100$ and $200 \mathrm{~mm})$. The robot was driven to the coordinate of the cylinder radius, as measured from the centre of the plate. The parts were built by rotating the table and maintaining the TIG head in the same orientation.

Prior to depositing material, the substrate plates were preheated by running the TIG torch three times over the substrate at a current of $140 \mathrm{~A}$, and the same travel speed as the experiment to be performed.

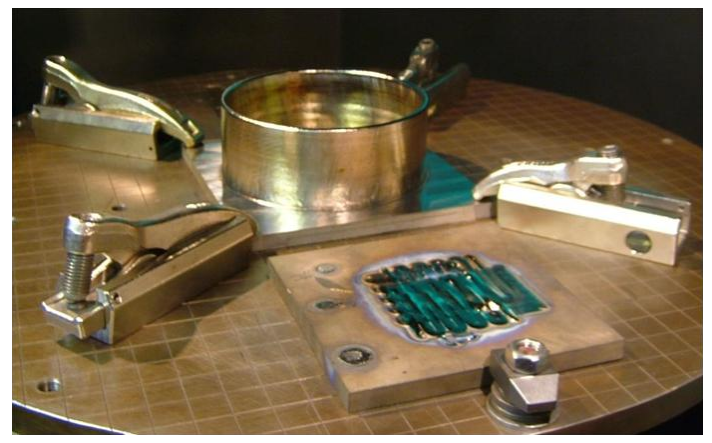

Figure 1 Experimental set-up

The deposition parameters, component dimensions and the response variables are given in Table 1. The height of the cylinders was $75 \mathrm{~mm}$. Part geometry (inner/outer diameter and part thickness), hardness and surface texture were measured as response variables for the experiment. Repeated measurements of part geometry were taken using a Vernier Calliper. Surface roughness measurements were taken according to ISO standard BS1134-1:1988; using a scanning length of 5.6mm with a cutoff of $0.8 \mathrm{~mm}$. The measurements were taken across the deposition layers. The measurements of hardness (Vickers) were taken using a Leitz micro-hardness tester with a load of $100 \mathrm{~g}$. An average of 40 indents was performed along the wall of each component. 
Table 1 Deposition parameters, the resulting geometry and response variables of the eight SMD components used for the development of the model (M1-M8) and of two components used for validation (V1-V2).

\begin{tabular}{|c|c|c|c|c|c|c|c|c|c|}
\hline & \multicolumn{3}{|c|}{ Deposition parameters } & \multicolumn{5}{c|}{ Responses } \\
\hline & $\begin{array}{c}\text { Energy } \\
\text { level } \\
\left(\mathrm{kJs} / \mathrm{mm}^{3}\right)\end{array}$ & $\begin{array}{c}\text { Travel } \\
\text { speed } \\
(\mathrm{m} / \mathrm{min})\end{array}$ & $\begin{array}{c}\text { Step } \\
\text { size } \\
(\mathrm{mm})\end{array}$ & $\begin{array}{c}\text { Progr. } \\
\text { diameter } \\
(\mathrm{mm})\end{array}$ & $\begin{array}{c}\text { Wall } \\
\text { thickness } \\
(\mathrm{mm})\end{array}$ & $\begin{array}{c}\text { Shrinkage } \\
(\%)\end{array}$ & $\begin{array}{c}\text { Height of } \\
\text { top region } \\
(\mathrm{mm})\end{array}$ & $\begin{array}{c}\mu \text {-Vickers } \\
\text { hardness } \\
(\mathrm{HV})\end{array}$ & $\begin{array}{c}\text { Surface } \\
\text { roughness } \\
(\mu \mathrm{m})\end{array}$ \\
\hline M1 & 34.76 & 0.40 & 1.0 & 100 & 4.86 & 8.90 & 7.44 & 343 & 4.18 \\
\hline M2 & 22.64 & 0.40 & 1.0 & 200 & 7.71 & 4.70 & 8.00 & 352 & 2.87 \\
\hline M3 & 1716 & 0.25 & 1.0 & 200 & 5.90 & 4.24 & 6.44 & 346 & 3.49 \\
\hline M4 & 11.06 & 0.40 & 0.5 & 200 & 5.00 & 3.91 & 4.82 & 347 & 2.98 \\
\hline M5 & 9.32 & 0.25 & 0.5 & 100 & 6.72 & 8.44 & 7.60 & 343 & 6.52 \\
\hline M6 & 15.26 & 0.25 & 0.5 & 200 & 9.90 & 4.31 & 8.6 & 346 & 4.65 \\
\hline M7 & 18.09 & 0.40 & 0.5 & 100 & 9.96 & 9.39 & 12.6 & 340 & 6.86 \\
\hline M8 & 33.68 & 0.25 & 1.0 & 100 & 11.57 & 9.05 & 13.0 & 340 & 4.58 \\
\hline V1 & 50.51 & 0.3 & 1.0 & 100 & 8.60 & 8.10 & 13 & 332 & 6.57 \\
\hline V2 & 47.20 & 0.3 & 1.0 & 100 & 10.20 & 9.00 & 13.6 & 334 & 4.58 \\
\hline
\end{tabular}

\subsection{Model development}

The problem of studying the behaviour of the SMD process was tackled using a design of experiments (DOE) approach [10]. By means of this statistical method, the factors that are thought to have an effect on the system's outputs can be systematically studied. The relationship between the factors and their effects can be determined using limited number of experiments. Thus, the main effects and their interactions can be calculated. A main effect is the effect of an independent variable averaging over all levels of the other independent variables in the experiment. An interaction between two factors is present when the effect of one factor depends on the level of the other factor.

Four factors were included in the experiment: programmed diameter, step size, travel speed and a parameter named 'energy level', $E$, that related the heat source power and the rate of raw material input. The energy level was calculated as Equation 1, where $\mathrm{T}$ is the arc current $[\mathrm{A}], \mathrm{V}$ the voltage $[\mathrm{V}], r$ the wire radius $[\mathrm{mm}]$ and $w$ the wire feed rate $[\mathrm{mm} / \mathrm{s}]$.

$$
E=\frac{T \times V}{\pi r^{2} w}
$$

The experimental design consisted of a one-half fractional factorial design with four factors at two levels (i.e. ${ }^{2_{\mathrm{IV}}^{4-1}}$ ). Table 1 provides the experimental factors with their corresponding levels. This DOE has a resolution IV, i.e. no main effect is aliased with any other main effect or with any twofactor interactions; however, two-factor interactions are aliased with each other.

Table 2 Experimental factors and their corresponding levels

\begin{tabular}{|l|c|c|}
\hline \multirow{2}{*}{\multicolumn{1}{|c|}{ Factor }} & \multicolumn{2}{c|}{ Level } \\
\cline { 2 - 3 } & Low & High \\
\hline Energy level $\left[\mathrm{kJs} / \mathrm{mm}^{3}\right]$ & 39.6 & 52.5 \\
\hline Travel speed $[\mathrm{m} / \mathrm{min}]$ & 0.25 & 0.40 \\
\hline Step size $[\mathrm{mm}]$ & 0.50 & 1.00 \\
\hline Programmed diameter $[\mathrm{mm}]$ & 100 & 200 \\
\hline
\end{tabular}

The DOE consisted of eight experimental observations; no replication was conducted. The experimental observations were fully randomised in order to minimise the effect of extraneous factors. Furthermore, randomisation assures the validity of the assumption that the observations are independent distributed random variables. No blocking was conducted.

The results of the analysis were used to evaluate the relative importance of the parameters and their interactions and to generate preliminary empirical process models that can be used to provide information for further work. 
An experimental matrix was generated using a DOE approach to examine the role of various process parameters on the SMD process (see Table 1). With this technique the number of experiments is streamlined and provides a systematic way to evaluate the influence of individual parameters and their interactions on the outputs.

Linear regression can be used to develop a predictive model based on the observed data relating system's outputs to systems parameters. The fitted model can be used to make predictions of the outputs. Linear regression analysis can also be used to quantify the strength of the relationship between system's outputs and parameters; to assess which parameter has no relationship with the responses.

The models were developed using the least squares approach based on multiple linear regression with five regressor variables (i.e. current (C), travel speed (ts), wire feed (wf), step size (Sz) and component diameter (D)). Four mathematical models of the form of equation (2) were developed, where $i=1$ to 4 (thickness, percent of shrinkage, surface texture and portion of fine Widmanstätten).

$$
y_{i}=\beta_{0}+\beta_{1 i} x_{C}+\beta_{2 i} x_{t s}+\beta_{3 i} x_{w f}+\beta_{4 i} x_{S z}+\beta_{5 i} x_{D}
$$

\section{Results and analysis}

\subsection{Wall thickness}

The experimental parts built range from 4.86 to $11.56 \mathrm{~mm}$ thick. The main effect plot for thickness, shown in Figure 2, suggests that the most significant parameter affecting the thickness of the walls is the energy level, followed by travel speed. Increasing the energy level will increase the size of the melting pool, thus increasing the width of the deposit. As the current increases, more material is needed to maintain the same arc gap. Wall thickness increases with increasing energy level and decreases with increasing travel speed and component size. Step size appears to have the smallest effect on wall thickness. None of the interactions between parameters had a significant effect. However the interaction between energy level and component size had a stronger effect than step size.

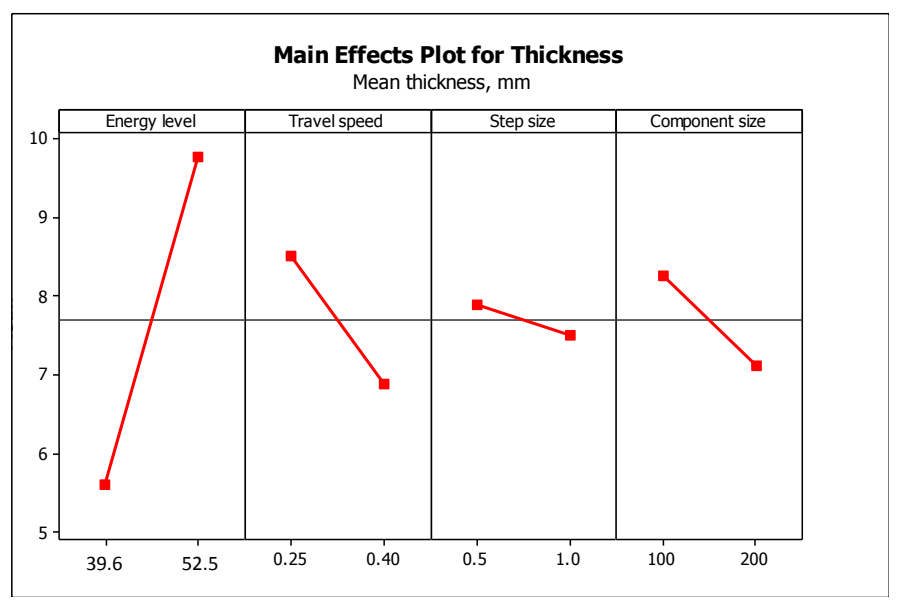

Figure 2 Main effect plot for thickness

\subsection{Shrinkage}

All experimental parts exhibited shrinkage; from a range of 3.9 to $9.4 \%$. Figure 3 shows the main effect plot for shrinkage \%. The factor that had the strongest effect was component size, with decreasing effect. This finding relates to the wall thickness results. Smaller parts have thicker walls, if the percentage of solidification is measured relative to the wall thickness, thicker parts will have a higher percent of shrinkage. No other factor or interaction had a significant effect. 


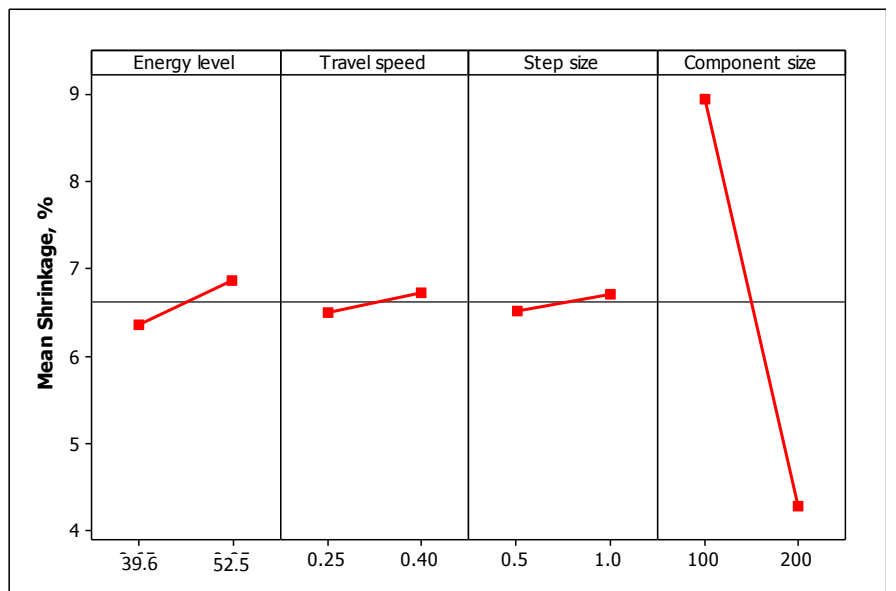

Figure 3 Main effect plot for Shrinkage \%

\subsection{Surface roughness $(R a)$}

The experimental parts had a range of surface roughness $(R a)$ of 2.9 to $6.9 \mu \mathrm{m}$. Figure 4 shows the main effect plot. Two factors had a strong effect on surface finish: energy level and step size. Surface roughness decreases with increasing energy level and decreasing step size. Figure 5 presents a plot of the relationship between surface roughness and current for different step sizes. Surface roughness was measured perpendicular to the deposition layers, hence capturing the difference in step size.



Figure 4 Main effect plot for Surface roughness Ra

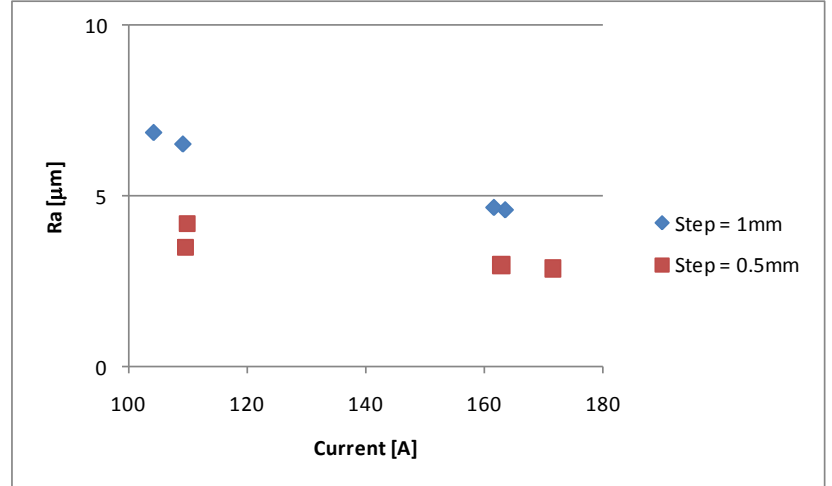

Figure 5 Relationship between surface roughness and current 


\subsection{Portion of finer Widmanstätten (height of top region)}

Some metallurgical aspects were also investigated. A typical cross-section of an SMD wall can be seen in Figure 6, where two different regions can be distinguished. This phenomenon has already been reported in [6]. The two regions are characterized by a Widmanstätten structure with different morphologies. The top region consists of finer lamella as a result of the last deposition layer experiencing temperatures high enough to be in the $\beta$ phase field. The microstructure in the bottom region has a coarser microstructure developed by the continuous SMD steps with temperatures within the two phase field $\alpha / \beta$. The height of the top region for all experimental parts was quantified (see Table 1).

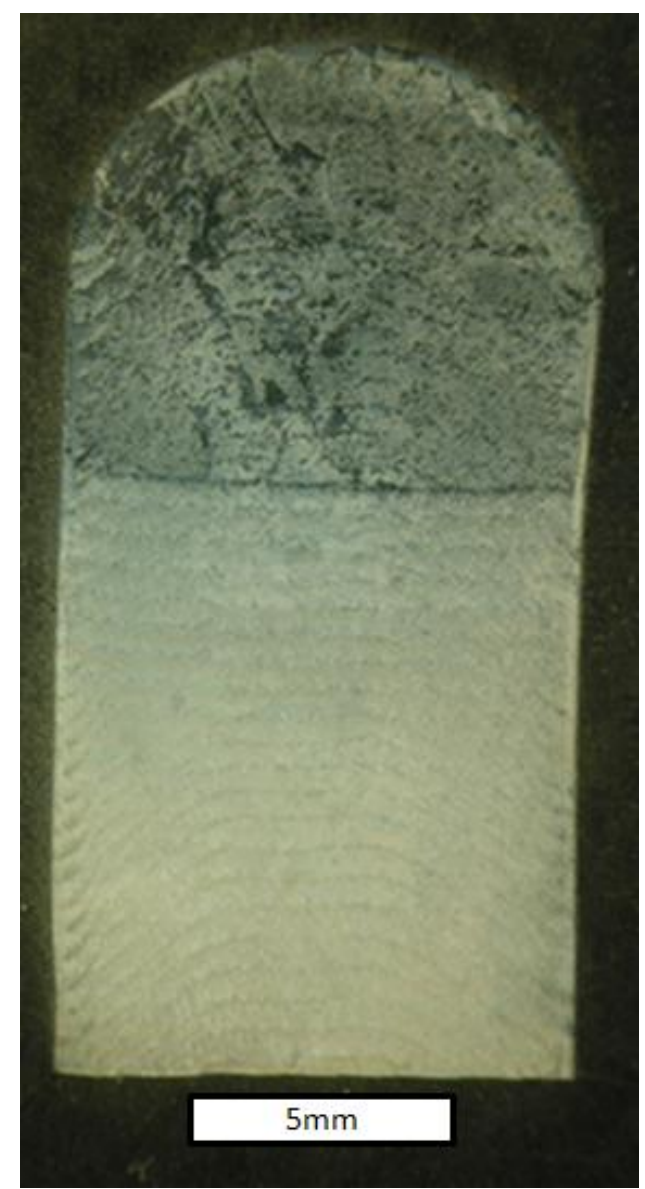

a)

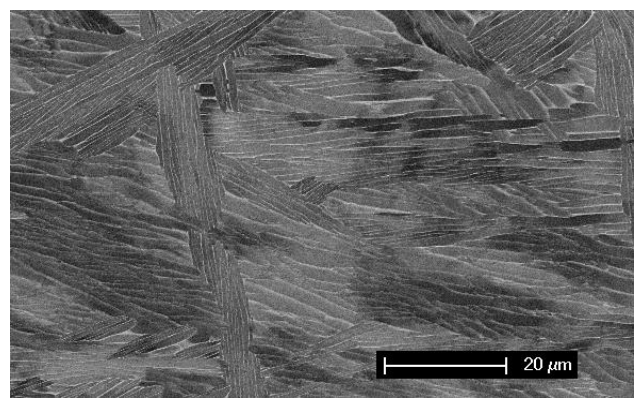

b)

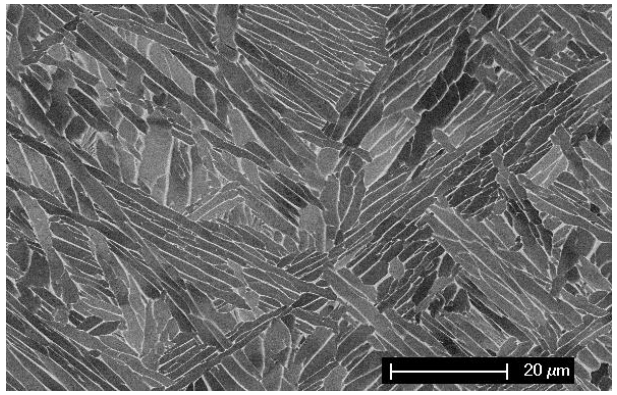

c)

Figure 6 Optical micrograph of etched cross-section (a) with insets of microstructure of top (b) and bottom (c) region

All the parts built presented the difference of microstructure characterised by two discernible regions. The regions ranged from 4,8 to 13,6mm height. Figure 7 presents the Main effect plot for the height of the top region. The factors that had a significant effect on the height of the top region were step size and travel speed. The height of the top region increased with step size and travel speed. Energy level did not have a strong effect; however the interaction between energy level and component size was strong. Even though the DOE analysis did not find energy level as having a strong effect on the height of the top region, the current and component size are significant factors. Figure 8 shows a graph of this relationship. The height of the top region increased with current and decreased with part diameter. 


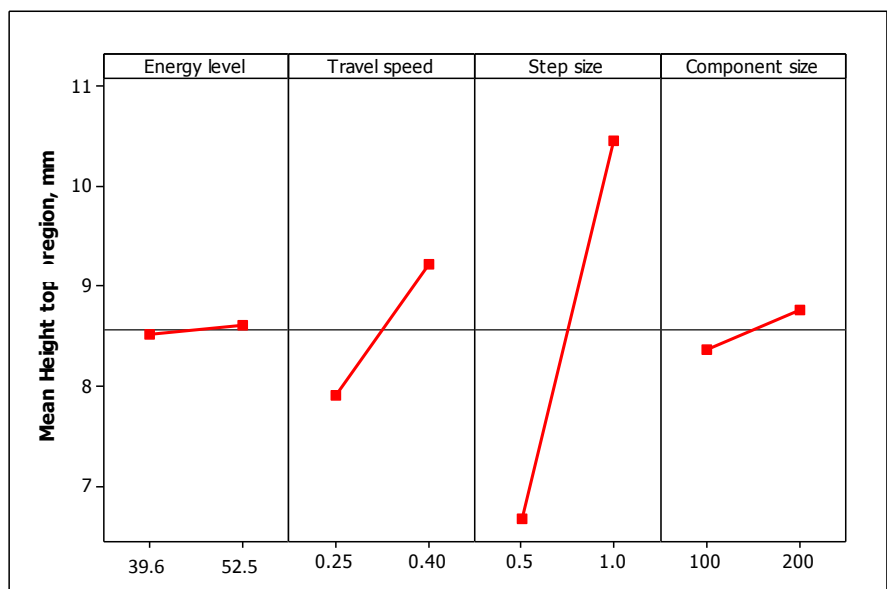

Figure 7 Main effect plot for Height of the top region

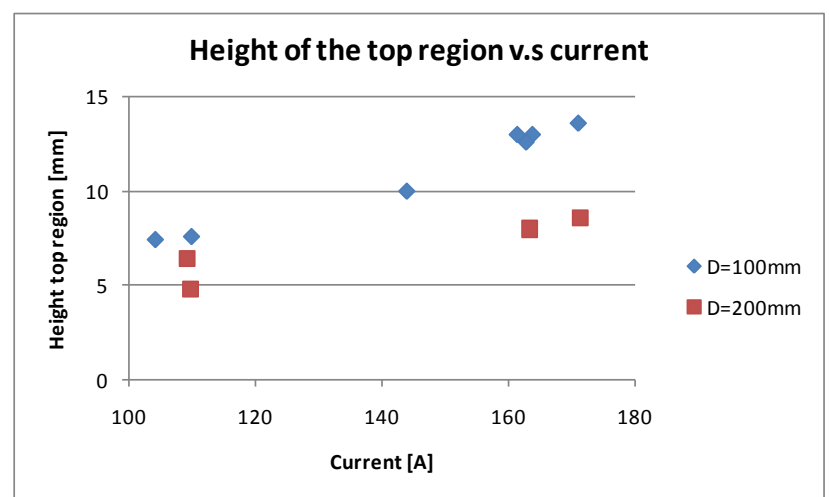

Figure 8 Relationship between current and height of the top region for different part diameters

\subsection{Micro hardness}

The mean hardness of the experimental parts was $341 \mathrm{HV}$ with a range of 325 to $352 \mathrm{HV}$ (see Table 1). No relationship was found for the parameters and range tested.

\section{Model Validation}

The models were validated using two previous builds. The parameters used to produce the parts were input into the models. The predicted shrinkage percentage, thickness, surface finish, height of the top region was compared to the experimental data. Table 3 produced a comparison of predicted versus experimental values. The model that had the maximum error was the surface finish one with $38.5 \%$; this may be due to the scatter of the data. The model predicted shrinkage with less than $11 \%$ error.

Table 3 Comparison of predicted versus experimental values

\begin{tabular}{|c|c|c|c|l|c|c|c|c|c|c|c|}
\hline \multicolumn{3}{|c|}{ Thickness } & \multicolumn{3}{c|}{ Shrinkage } & \multicolumn{3}{c|}{ Height } & \multicolumn{3}{c|}{ Surface finish } \\
\hline $\begin{array}{c}\text { Data } \\
(\mathrm{mm})\end{array}$ & $\begin{array}{l}\text { Model } \\
(\mathrm{mm})\end{array}$ & $\begin{array}{l}\text { Error } \\
(\%)\end{array}$ & Data & Model & Error & Data & Model & Error & Data & Model & Error \\
\hline 8.6 & 9.96 & 13.64 & 8.10 & 9.07 & 10.70 & 13.0 & 11.90 & 9.24 & 6.57 & 4.74 & 38.52 \\
\hline 10.2 & 10.8 & 5.56 & 9.00 & 9.02 & 0.22 & 13.6 & 12.56 & 8.29 & 10.12 & 4.16 & 10.13 \\
\hline
\end{tabular}

\section{Conclusions and further research}

In this work a study based on a design of experiments was carried out to evaluate the relative significance of SMD input parameters. Four parameters were studied (energy level, travel speed, step size and component diameter) on a fractional factorial DOE. The energy level was calculated as a ratio of heat source power and the rate of raw material input. This approach was used to develop empirical 
relationships to systems outputs and preliminary models for predicting part thickness, shrinkage percentage, portion of finer Widmanstätten and surface finish.

The results of the analysis showed that the four factors investigated had a strong effect on system's outputs. The energy level had a strong effect on wall thickness and part surface finish. Travel speed is an important factor influencing part thickness and portion of finer Widmanstätten. Step height is a key process variable for surface finish and portion of finer Widmanstätten. Finally, component size has a strong influence in percentage of shrinkage.

The preliminary empirical models developed had a mean relative error of less than $10 \%$. The models can be improved by incorporating more data into the analysis. The current models were developed using a fractional factorial design with no centre point. The model would benefit from incorporating centre points, replicates and the data from the complementary design points.

The model could also be extended to relate more variables to the experimental data. If measurements of temperature could be taken, the methodology could be used to relate temperature history to the time temperature transformation diagram to help predict component microstructure.

\section{Acknowledgement}

The research presented here was carried out within the RAPOLAC project under contract number 030953 of the 6th Framework Programme of the European Commission (www.RAPOLAC.eu). The authors would like to express their gratitude to Garry Hibbert and Dave Spafford for producing the SMD builds.

\section{References}

1. Smallman, R.E. and R.J. Bishop, Modern physical metallurgy and materials engineering: science, proces, applications. 6th ed. ed. 1999, Oxford: Butterworth-Heinemann. 438.

2. Boyer, R.R., An overview on the use of titanium in the areospace industry. Materials Science and Engineering: A, 1996. 213(1-2): p. 103-114.

3. Kruth, J.P., M.C. Leu, and T. Nakagawa, Progress in Additive Manufacturing and Rapid Prototyping. CIRP Annals - Manufacturing Technology, 1998. 47(2): p. 525-540.

4. Chivel, Y.A., Laser-assisted micro-and nanotechnologies 2003. Proceedings of the Society of Photo-optical instrumentation engineers, 2004. 5399: p. 228-233.

5. Paul, C.P., et al., Investigating laser rapid manufacturing for Inconel-625 components. Optics \& Laser Technology, 2007(39): p. 800-805.

6. Baufeld, B., O.v.d. Biest, and R. Gault, Microstructure of Ti-6Al-4V specimens produced by Shaped Metal Deposition. Int. J. Mat. Res., 2009. 100: p. 1536-1542.

7. Clark, D., M. Bache, and M. Whittaker, Shaped metal deposition of a nickel alloy for aero engine applications. J. Mater. Process. Technol., 2008. 203: p. 439-448.

8. Wallace, T.A., et al. A Design of Experiments Approach Defining the Relationships Between Processing and Microstructure for Ti-6Al-4V. in 15th Solid Freeform Fabrication Symposium, 2-4 Aug. 2004, Austin, TX, United States 2004.

9. Baufeld, B. and O. van der Biest, Mechanical properties of Ti-6Al-4V specimens produced by shaped metal deposition. Science and Technology of Advanced Materials, 2009. 10(1).

10. Montgomery, D.C., Design and analysis of experiments. 2000: Chichester: Wiley. 643. 